\title{
International workshop on molecular genetics of haemochromatosis, held at Villa Feltrinelli, Gargnano (Bs), Italy, 25 September 1994
}

In terms of medical genetics haemochromatosis is an old and stubborn disorder. It was first described in the 19th century but it was Sheldon ${ }^{1}$ who in 1935 reviewed more than 300 cases and made many proposals about the nature of the disorder as well as suggesting that it was an inherited defect. Haemochromatosis has been actively investigated over the last $\mathbf{5 0}$ years and for nearly 20 years we have had a reasonably accurate genetic localisation, within $1 \mathrm{cM}$ of HLA-A on chromosome $6 \mathrm{p}$. It is one of the most common inherited disorders in European populations and can be treated effectively if the diagnosis is made early. However, progress in identifying the gene (HFE) remains painfully slow. Why is this? This International Workshop has shown that it is not because of lack of interest or application and has also provided some clues as to why identifying the gene is proving so difficult. The meeting was organised by Clara Camaschella (Torino), Bruno Dallapiccola (Roma/San Giovanni R), Giorgio De Sandre (Verona), Paolo Gasparini (San Giovanni R), and Alberto Piperno (Milano) and supported by Università di Milano, Università di Torino, Università di Verona, CNR-CIOS (Torino), and IRCCSOspedale "Casa Sollievo della Sofferenza".

The topic was introduced by Professor Laurie Powell (Brisbane). He summarised our knowledge of haemochromatosis and stressed the frequency of the disorder in a number of populations of European origin, in which more than 1 in 12 are heterozygous for haemochromatosis. He proposed that the HLA linked, inherited, parenchymal, iron storage disease should be called simply "haemochromatosis" and that this term is not used for secondary iron loading disorders such as thalassaemia major, sideroblastic anaemia, and the non-HLA linked South African siderosis. We still do not know whether the excessive iron absorption, which appears to result from a normal uptake of iron by the mucosal cells and an increased transfer to the plasma, is a primary defect or secondary to a defect elsewhere in the body. He stressed the importance of determining the iron concentration in the liver and expressing this as the hepatic iron index (related to age) for accurate diagnosis. HLA typing is essential for the identification of other homozygotes within a family. The progression of iron loading with age in homozygous subjects has been well documented and this does not appear to occur in heterozygotes. The clinical message is still that early diagnosis and treatment can provide a normal life expectancy.

In genetic terms the history of haemochromatosis is a little unusual. We still have no chromosomal translocations or informative recombinations to provide a more precise gene location than the long established "within $1 \mathrm{cM}$ of HLA-A". A brief consideration of the various methods of analysis which have been used then led up to the major theme of the first session, allelic association studies.

\section{First session: positional cloning}

E Jazwinska (Brisbane) described studies of linkage, linkage disequilibrium, and haplotype analysis in Australian patients, using markers from D6S248 (between HLA-B and -A) to D6S105 (a microsatellite marker $3 \mathrm{cM}$ telomeric to HLA-A). She concluded that the candidate region for the gene extends between D6S248 and D6S105. D6S105 showed the strongest linkage disequilibrium with the gene ${ }^{2}$ and haplotype analysis showed the most likely location for the gene was telomeric of HLA-A between HLA-F and D6S105.

$\mathrm{J}$ Yaouanq (Rennes) produced data on a large series of normal and haemochromatosis chromosomes from Brittany, adding to the published data ${ }^{3}$ on the analysis of marker D6S105. Disequilibrium with the HFE gene increased from 1.82 (100 kb centromeric to HLA-A) to HLA-A, fell to HLA-F, and then increased again at D6S105.

R Raha-Chowdhury (Cardiff) described allelic association studies for loci from HLA-B to D6S299 which is approximately $4 \mathrm{cM}$ from HLA-A towards the telomere. Using a highly informative polymorphism for the HLA-F $5^{\prime}$ untranslated region there were equally strong associations for alleles at HLA-A, HLA-F, and D6S105. This contrasted with previous studies ${ }^{3}$ suggesting that HLA-F represented the telomeric limit for the candidate gene region. Alleles at D6S299 showed no association with haemochromatosis and this marker provided a new telomeric limit.

C Camaschella (Torino) presented data for the Italian population. The analysis included two new markers near HLA-A (Y39 and Y52). All the region between HLA-A and D6S105 was found to be in linkage disequilibrium but the strongest association was found with Y52.

$M$ Worwood (Cardiff) examined allelic associations in unrelated patients and controls (blood donors) confirming the strong association $\mathrm{s}^{4}$ between haemochromatosis and 
D6S265 (close to HLA-A) and D6S105. Markers towards the telomere such as D6S299 and D6S276 showed no association with haemochromatosis, neither did D6S273 which may be centromeric to HLA-A. D6S306, 258, and 464 all had alleles which were more common in haemochromatosis but these alleles were also very common in controls.

All the speakers reported a common haplotype characterised by D6S265-1, HLA-A3, and D6S105-8. It accounts for 48 to $50 \%$ of HFE chromosomes in France and the UK and for approximately $30 \%$ in Australia and Italy. It is present at low frequency or even absent in normal chromosomes. It could represent the ancestral HFE haplotype carrying the gene for haemochromatosis.

The general discussion focused on the problems of diagnosis of haemochromatosis, differences in allele frequencies at the various loci, and the selection of normal controls. There was a consensus that use of normal subjects as controls in haplotype studies may be misleading. If about $10 \%$ of the population carry the haemochromatosis gene then the only acceptable way of examining normal chromosomes will be to use "non-haemochromatosis" chromosomes selected from pedigrees with the haemochromatosis gene. There were clearly different views about the location of the haemochromatosis gene, either close to HLA-A or close to D6S105. However, Le Gall (Rennes) considered that the explanation may be that there are two types of "stable" chromosomes. There are those carrying the B8:A1: 105-8 haplotype, commonly found in northern European populations, and in haemochromatosis there is the A3:105-8 haplotype. If the genetic distance between HLA-A and D6S105 is estimated to be 2 to $3 \mathrm{cM}$ in normal populations, it may be that the lack of recombination in the "stable" haplotype found in haemochromatosis provides the reason for the close association of both HLA-A and D6S105 with haemochromatosis.

\section{Second session: physical mapping of the candidate(s) region(s)}

Here progress in analysing part of the candidate region for HFE was reported. J Gruen (New Haven) began the session by giving a brief history of the mapping of the HLA region, particularly the class I region. He described the use of radiation hybrids for deletion mapping, pulse field gel electrophoresis, and recent studies using YACs. He described a complete contig of CEPH YACs for the HLA-A class I region. A number of haplotypes are associated with deletions of from 50 to $90 \mathrm{~kb}$ and there is considerable heterogeneity of NotI fragments on pulse field gel electrophoresis. The A31 haplotype has additional DNA.

A Volz (Berlin) then described his analysis of the chromosomal region between the HLA complex and the histone gene cluster using radiation hybrids. A number of $\mathrm{D}$ segments, including D6S299, D6S105, D6S148, D6S248, D6S131E, D6S258, and D6S265, were examined along with six $\mathrm{H} 1$-histone genes. Methodology also included FISH and long range restriction mapping. He calculated that this region extends for at least $9.5 \mathrm{Mb}$ and that the minimum distance between HLA-A and D6S105 is approximately $3100 \mathrm{~kb}$. From D6S105 to histone gene H1F3 appeared to be a minimum of $2600 \mathrm{~kb}$. Since so far no real physical linkage between HLA-F and D6S105 has been found he concluded that the haemochromatosis gene may be more remote from HLA-A than previously thought. ${ }^{5}$

C Stone (Oxford) showed by FISH that D6S105 is telomeric to HLA-A (at least 1 to $2 \mathrm{Mb}$ ). Analysing YACs containing D6S105 they produced three new CA repeats from this region: CS2 (identical to the Genethon microsatellite D6S464), CS3, and CS4. Linkage disequilibrium analysis in haemochromatosis patients indicated that both CS2 and CS4 are associated with haemochromatosis, although to a lesser extent than D6S105. This was because the most frequent alleles in haemochromatosis patients are also the most frequent in normal populations. ${ }^{6}$

P Gasparini (San Giovanni Rotondo) presented information on new polymorphisms and markers potentially useful for linkage studies in haemochromatosis. They were obtained from CEPH YACs 225B1 and 521F7 encompassing the HLA class I region. Using PCR protocols based on primers for different target sequences such as Alu repeats, splicing sites, and microsatellites, 20 new fragments (four polymorphic) were identified; two of them (Y39 and Y52) were useful for linkage analysis. ${ }^{7}$

$M$ Burt (Brisbane) then presented a physical map of the HLA-A region using YACs from the CEPH libraries. Results were confirmed by FISH. D6S265 was localised just centromeric to HLA-A at a maximum of $70 \mathrm{~kb}$ from HLAA. D6S248 was also shown to be centromeric of HLA-A. So far it has not been possible to link YACs containing HLA-A and D6S105.

L Pichon (Rennes) described a complete physical map of four YACs encompassing the HLA-A/HLA-F subregion and the structural organisation of all the multiple families described in this region (HLA-A, HCGII, HCGIV, P5, and pMC6.7). This group is systematically sequencing all the DNA area between HLA-A and HLA-F (now available in cosmid contigs) in order to isolate all the genes.

The studies reported indicate the problems encountered in the physical analysis of the HLA class I region, particularly restriction polymorphisms, differences in fragment length according to the haplotype, expansion and construction of the region, and presence of multigenic families.

\section{Third session: candidate genes}

$\mathrm{V}$ David reported on the seven candidate genes obtained from YAC B $30{ }^{8}$ Since most of the cDNAs isolated from the small intestinal mucosa were truncated in their $5^{\prime}$ ends, several other cDNA libraries were screened. Among these cDNAs, one is homologous to the yeast suppressor sup gene and might be involved in translational fidelity. The subcloning of another 
YAC (168E5) allowed the isolation of four new expressed and conserved sequences. She emphasised that there are a number of small genes around HLA-A and several multigenic sequences in the region between HLA-A and F.

J Gruen (New Haven) described the isolation of new non-HLA gene fragments from $6 \mathrm{p} 21.3$ by cDNA hybridisation selection. ${ }^{9} \mathrm{He}$ presented evidence about AFP, a zinc finger protein. This has been located on YAC B30H3 and produces a transcribed fragment which is rich in cystine and histidine residues. It may correspond to David's clone number 2 and because of its centromeric location he did not consider it to be a good candidate.

M Chorney (Hershey) discussed expansion and contraction in the HLA-A region, in particular examining coding sequences from HLAA to HLA-F with emphasis on duplications of the major class I locus detected by pMC6.7, a probe from the HLA-H subregion between HLA-A and HLA-F. ${ }^{10}$ This cross reacts with five fragments in the class I region. In subjects with HLA-A24 there is a deletion of one of these fragments and in those carrying A31 there is a loss of another fragment with two additional fragments appearing. cDNA screening with pMC6.7 has detected two cDNAs both of which display multiple transcripts. This is clearly a region in which considerable gene duplication has occurred.

J Rommens (Toronto) described a gene tracking approach using the CEPH YACs $225 \mathrm{~B} 1$ and $421 \mathrm{~F} 7$. The immobilised YAC is hybridised to cDNA from several tissues, including the intestinal cell line $\mathrm{CACO} 2$, after treatment to prevent hybridisation of repeat sequences. Further steps will involve hybridisation to mRNA from more tissues, mapping, and sequence analysis.

J Drysdale (Boston) considered possible candidate genes that might account for anomalies in iron metabolism in haemochromatosis. These included a ferritin receptor, a ferrireductase, an iron transporter, and a new sequence which may encode a secreted ferritin and is represented in a distinct multigene family with members on chromosome 6 (in collaboration with Drs A Walker and A Monaco).

The studies reported in this session strengthen the view that in the absence of any candidate genes identified in terms of function, progress in identifying the gene must depend on reducing the size of the candidate region.

\section{Fourth session: haplotype-phenotype correlations}

The fourth session was an attempt to seek associations between particular haplotypes and phenotypic expression. E Jazwinska discussed the contribution of genetics to the clinical phenotype in Australian patients through three studies: (1) examination of the phenotype of affected sib pairs, (2) analysis of biochemical indices of heterozygotes, and (3) haplotypephenotype correlation in patients. Serum ferritin, transferrin saturation, and liver concentration were similar in sib pairs indicating that environmental factors have smaller impact than genetic background. Comparing 290 heterozygotes with 431 normal subjects she showed that the mean transferrin saturation was significantly higher in heterozygotes $(35 \%)$ than in normal controls (25\%). Patients with two copies of the common haplotype (A3, D6S265-1, D6S105-8) had significantly higher hepatic iron indices than the other patients.

A Piperno (Milano) described some preliminary data on gentotype/phenotype in Italian patients. He found that the ancestral haplotype is associated with a relatively homogeneous, severe expression of the disease. He also found concordance in the expression of the disorder (hepatic iron index or amount of iron removed by phlebotomies/age) in sib pairs and in patients showing partially identical (HLA-A:D6S265: D6S105) haplotypes.

Finally, T Rouault (Bethesda) summarised new understanding about the so-called African iron overload, a non-HLA linked disorder in which a genetic predisposition cooperates with a high iron diet. She found that external factors are important: those subjects having a high iron diet have higher transferrin saturations than subjects with a genetic predisposition who are on a diet of normal iron content.

\section{Conclusions}

There was general agreement that the workshop had been extremely valuable in focusing on haemochromatosis in the context of current methods of genetic analysis. The meeting concluded with a proposal for international collaboration to accelerate progress in locating the gene.

(1) It was agreed that the search for chromosome rearrangements and recombinants in this region is of vital importance but requires accurate assessment of iron status within families.

(2) More informative markers around D6S105 are required.

(3) Haplotype analysis should be carried out on normal and haemochromatosis chromosomes with such markers. Comparison of data from different populations should help to localise the gene.

(4) It was agreed that rapid dissemination of results would prevent wasteful duplication and that a newsletter be set up through email.

(5) It was agreed that a further informal meeting should be arranged to take place within one year.

MARK WORWOOD

Department of Haematology, University of Wales College of Medicine, Heath Park, Cardiff CF4 4XN, UK PAOLO GASPARINI

Servizio di Genetica Medica, IRCCS Ospedale "CSS", 71013 San Giovanni Rotondo (FG), Italy

CLARA CAMASCHELLA

Dipartimento di Scienze Biomediche e Oncologia Umana, Sezione Clinica, CNR-CIOS Ospedale San Luigi Gonzaga, 10043 Orbassano, Torino, 
1 Sheldon JH. Haemochromatosis, London: Oxford University Press, 1935.

2 Jazwinska EC, Lee SC, Webb SI, Halliday JW, Powell LW. Localization of the hemochromatosis gene close to D6S105. Am f Hum Genet 1993;53:347-52.

3 Yaouanq J, Perichon $\mathrm{M}$, Chorney $\mathrm{M}$, et al. Anonymous marker loci within $400 \mathrm{~kb}$ of HLA-A generate haplotypes in linkage disequilibrium with the hemochromatosis gene in linkage disequilibrium with the hemoch
(HFE). Am f Hum Genet 1994;54:252-63.

4 Worwood M, Raha-Chowdhury R, Dorak MT, Darke C, Bowen DJ, Burnett AK. Alleles at D6S265 and D6S105 define a haemochromatosis-specific genotype. $\mathrm{Br} f$ Haematol 1994;86:863-6.

Volz A, Boyle JM, Cann HM, Cottingham RW, Orr HT, Ziegler A. Report of the second workshop on human

6 Stone C, Pointon JJ, Jazwinska EC, et al. Isolation of CA dinucleotide repeats close to D6S105; linkage disequilibrium with haemochromatosis. Hum Molec Genet 1994;3:2043-6.

7 Totaro A, Grifa A, Roetto A, et al. New polymorphisms and markers in the HLA class I region: relevance to the hereditary hemochromatosis. Hum Genet (in press).

8 El Kahloun A, Chauvel B, Mauvieux V, et al. Localization of seven new genes around the HLA-A locus. Hum Molec of seven 1993;2:55-60.

9 Goei VL, Parimoo S, Capossela A, Chu TW, Gruen JR Isolation of novel non HLA gene fragments from the Isolation of novel non HLA gene fragments from the hemochromatosis region (6p21.3) by cDNA hy

10 Venditti CP, Harris JM, Geraughty DE, Chorney MJ. Mapping and characterization on non-HLA multigene assemblages in the human MHC class I region. Genomics 1994;22:257-66. 